\title{
El plan de medios: principales respuestas a la eficacia publicitaria ${ }^{1}$
}

Natalia Papí Gálvez | natalia.p@ua.es

UNIVERSIDAD DE ALICANTE

Resumen: El artículo expone el desarrollo de dos conceptos fundamentales de la planificación (la cobertura y la frecuencia) como fruto de la reflexión sobre la contribución de los mismos a la eficacia de la publicidad. Se identifican tres niveles: la maximización de la cobertura o de la frecuencia del plan de medios; la optimización de estos criterios en busca de una respuesta positiva; y el abandono de la modelización basada en un hipotético soporte medio para enfocar la atención en el efecto del anuncio.

Palabras clave: planificación de medios publicitarios, eficacia publicitaria, cobertura, frecuencia

Abstract: This article outlines the evolution of two key concepts of planning (reach and frequency) as a result of reflection on advertising effectiveness. It outlines three levels: maximization of reach or frequency, optimization of these criteria on the basis of a positive response, and replacement of the hypothetical adverage support by the effect of advertising.

Keywords: media planning, advertising effectiveness, reach, frecuency

\footnotetext{
${ }^{1}$ Inserto en el proyecto Indicadores sociales en comunicación para la igualdad de género: herramientas para la evaluación de las políticas de comunicación, del plan nacional I+D+I subvencionado por el Instituto de la Mujer (ref. 125/02). El objetivo principal de este proyecto es proponer un sistema de medición para analizar la difusión y el conocimiento de las políticas de igualdad de género en materia de conciliación a nivel nacional. Para ello se plantea si la medición de la eficicacia de las campañas institucionales se puede realizar a través de los mismos criterios que se establecen en la publicidad comercial, lo que conduce a la revisión y síntesis de los fundamentos.
} 


\section{Introducción: La eficacia publicitaria y la planificación de medios}

\subsection{Medición de efectos}

Desde la segunda mitad del siglo pasado, la planificación de medios publicitarios ha crecido como disciplina y se ha consolidado como área de trabajo en publicidad. En todo este periodo ha desarrollando un lenguaje propio, establecido criterios y proporcionado procedimientos para la estimación de tales criterios. Ha generado, en definitiva, conceptos que se han consolidado como los fundamentos de la disciplina. Este artículo destaca aquellos que son clave para la planificación de medios y muestra su evolución en términos de la respuesta a la eficacia publicitaria.

Se suele entender por eficacia publicitaria el grado de consecución de los objetivos marcados para la publicidad. Los estudios realizados en este campo no conducen a resultados unívocos pues dependerá justamente de lo que se pretende conseguir para establecer el grado de eficacia del anuncio.

La investigación sobre eficacia proporciona conceptos y herramientas de análisis que ayudan a medir los resultados obtenidos tras el lanzamiento de una campaña. Algunos autores (Beerli, 1999; Martín, 1999) ordenan las técnicas de medición de la eficacia de acuerdo con los efectos que pudiera generar la publicidad en tres grupos: aquellas centradas en la medición de la atención, comprensión y recuerdo del mensaje (las llamadas técnicas cognitivas), las que se encargan de medir las actitudes (las afectivas) y las que atienden a las conductas (las conativas). La dificultad para provocar el efecto deseado, y alcanzar el objetivo, crece en cada grupo. El recuerdo de la campaña es un objetivo siempre presente en la comunicación publicitaria aunque no se haga explícito. Y aunque algunos autores destacan la necesidad de que, en última instancia, la publicidad deba responder a las ventas (González y Carrero, 2003; Martínez, 1996) existen limitaciones a la hora de establecer una relación directa y perfecta entre la comunicación publicitaria y el cambio de conducta del consumidor.

La planificación de medios publicitarios contribuye a la eficacia sólo desde uno de los ángulos posibles pues se trata de un concepto complejo que comprende otros elementos relacionados, por ejemplo, con la elaboración del mensaje. En concreto, la contribución de la planificación de los medios se circunscribe básicamente en dos tareas, pilares de la actividad profesional del planificador: la correcta selección de medios y soportes de acuerdo con el público objetivo; y el acierto de la frecuencia de los impactos a lo largo del tiempo dada una campaña. Estas dos tareas ayudan fundamentalmente a conseguir atención y recuerdo, aunque si se toman las decisiones equivocadas podrían repercutir negativamente generando rechazo hacia la campaña y, por tanto, una actitud desfavorable (León, 1996). 
1.2. La relación entre la frecuencia de exposición y el recuerdo: antecedentes e ideas clave

El recuerdo toma especial protagonismo cuando se profundiza en las respuestas clásicas a la eficacia desde la planificación. Se sabe que mantener el recuerdo de una campaña publicitara no sólo se rige por el número de exposiciones (repetición) sino también por el intervalo temporal que media entre estas exposiciones. En realidad, estas dos consideraciones son las que pautan todo el desarrollo conceptual que ha experimentado la planificación de medios publicitarios en su contribución a la eficacia publicitaria.

Los estudios que se centran en la acumulación del recuerdo (y de la atención) a través de la repetición se remontan a finales del siglo XIX, incluso relacionados con la publicidad. Todos los estudios pioneros que trataron de relacionar el recuerdo con las campañas publicitarias conducían a cuatro conclusiones claras:

\footnotetext{
- A más repetición, mayor recuerdo. - El recuerdo disminuye inmediatamente después de la exposición al mensaje, pero el olvido se atenúa progresivamente. Hay siempre un residuo de recuerdo. - La asociación, comprensión y ritmo ayudan fuertemente a la memoria. • Lo que se aprende rápidamente se recuerda mejor que lo que se aprende lentamente (Díez y Martín, 1999: 374)
}

Los autores anteriores a Krugman, que también han marcado el desarrollo posterior del análisis de la frecuencia de exposición en planificación de medios (autores como Zielske, Ray y Sawyer o la agencia McCollum/Spielman) corroboran, aunque matizan, las conclusiones de Díez y Martín (1999). Estas investigaciones introducen otros factores que pueden estar afectando al recuerdo como es el tipo de producto, el tipo de mensaje transmitido y el medio.

Sin duda, la mayor contribución a los estudios de la frecuencia de exposición en relación con su capacidad para generar memoria es proporcionada por la ley de recuerdo de Morgensztern. Esta ley permite relacionar los estímulos publicitarios con la memorización. Se basa en la respuesta del individuo al estímulo (el anuncio) en términos de recuerdo que adquiere un valor entre el 0 (ningún efecto) y el 1 (se recuerda completamente). Esta respuesta tiene un concepto propio cuando es aplicado a la publicidad: el efecto publicitario.

La expresión matemática de la ley es $E_{n}=1-(1-\beta)^{n}$; donde $E_{n}$ es el efecto publicitario con un número determinado de inserciones y $ß$ es un coeficiente que representa el desgaste ( $u$ olvido) de la memoria a medida que pasa el tiempo y el efecto producido con cada nuevo contacto. Así, el coeficiente ß es un valor que se ajusta a una campaña concreta, con un producto determinado. El gráfico proporcionado por la ley de recuerdo es llamado "curva de respuesta" y representa la frecuencia que se considera ideal (Martínez, 1992).

La función de recuerdo conduce a curvas asintóticas (ver ejemplo en la figura 1a) por las que se deduce que la relación entre el número de contactos y el recuerdo responde a dos características (Díez y Martín, 1999): 
- Por un lado, a medida que se incrementan los contactos el efecto conseguido, sea con el medio que sea, tiende a ser total (alcanza como valor la unidad). Es decir, a medida que una persona se expone al estímulo tiende a recordar el anuncio completamente.

- Por otro lado, se observa cómo los primeros contactos consiguen un efecto elevado, pero posteriormente el efecto va decreciendo. En consecuencia, desde un punto de vista económico, llegará un momento en el que un nuevo contacto no proporcionará un incremento rentable de recuerdo. En este caso, es preciso fijar un umbral máximo por razones económicas pero también lo será por el posible cansancio que genera la repetición excesiva del anuncio, cuyos efectos pueden ser contraproducentes a los objetivos perseguidos.

\section{a.- curva asíntota}

Efecto

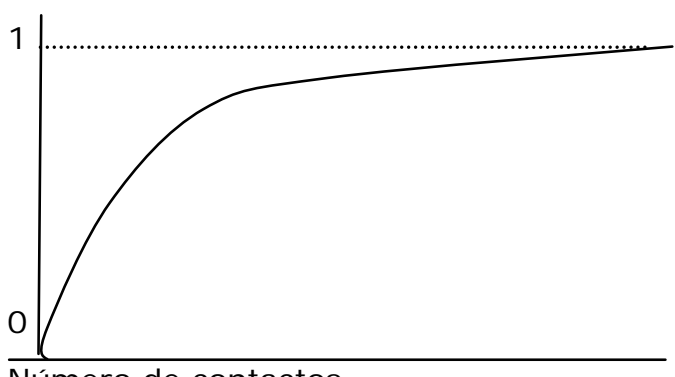

Número de contactos b.- representación simbólica recuerdo-atención

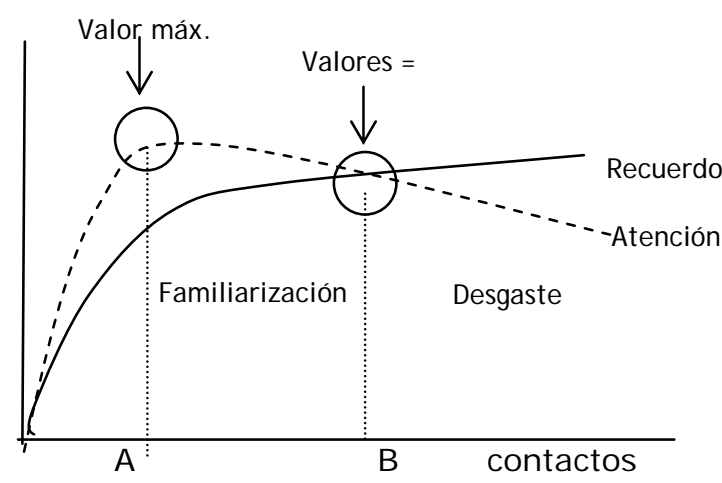

Fuente: Elaboración propia a partir de los datos proporcionados por Díez y Martín (1999) y Martínez (1992)

Figura 1. Curva de la ley de recuerdo (a.-) y representación de la relación recuerdo-atención con umbrales mínimos y máximos (b.-)

Además, Armand Morgensztern, en el medio impreso, observó una relación entre el tiempo dedicado a la atención del anuncio y la generación de recuerdo del mismo con el nivel de exposiciones. La gráfica (ver figura 1b) trata de representar, de forma algo rudimentaria pero sencilla, las siguientes ideas (Díez y Martín, 1999):

- En los primeros contactos con el anuncio, el tiempo de atención se dedica a conseguir tasa de recuerdo publicitario. En esta situación se incrementa más el tiempo de atención que la tasa de recuerdo.

- Los contactos posteriores hacen que el individuo se familiarice con el anuncio. El incremento del recuerdo es mayor que el aumento de la atención.

- El número mínimo de impactos es el valor que hace máximo el tiempo de atención (representado como A en la gráfica). El número máximo será aquel valor en el que atención y recuerdo son iguales (representado como B en la gráfica). 
Si se atiende al gráfico (figura la) de la ley de recuerdo se observa que será preciso un número de contactos determinado para alcanzar el efecto total (el valor 1) que no tiene porqué corresponder con el valor B representado como umbral máximo en la representación recuerdo-atención.

En este punto, surgen algunas limitaciones de la mano del tipo de atención y su capacidad para generar recuerdo (i.e. Del Río, 2000) pues la relación no parece ser tan directa cuando se observa en condiciones habituales en lugar de las que se suelen generar en laboratorio. Ésta es sólo una de las críticas a las teorías que tratan de encontrar una respuesta unívoca a la eficacia desde la planificación.

\section{Evolución de los conceptos de cobertura y de frecuencia}

A la luz de las anteriores consideraciones, se pueden identificar, a grandes rasgos, los siguientes tres niveles que reflejan la evolución de dos conceptos fundamentales (cobertura y frecuencia) en virtud de su capacidad para responder a la eficacia de la publicidad:

- En un primer nivel se ubica la cobertura como principal protagonista. En esta etapa, por la década de los 60, la cobertura es objeto de estudio de muchas propuestas preocupadas por su modelización. Unida a la cobertura se debe tener en cuenta la frecuencia. Ambos criterios, junto con el presupuesto, marcan la estrategia publicitaria en materia de medios.

- Un segundo nivel estaría formado por los criterios de alcance y de frecuencia efectiva como fruto de la reflexión en torno a la idoneidad de los anteriores conceptos y estrechamente relacionados con la búsqueda de niveles de exposición apropiados para conseguir una respuesta favorable al estímulo publicitario. Por tanto, la cobertura y frecuencia efectiva toman sentido tras el desarrollo teórico relacionado con los umbrales de exposición para conseguir recuerdo de mediados de la década de los 70.

- Finalmente, un tercer nivel surge de los avances y de las limitaciones reales de la puesta en práctica de los anteriores criterios. Así, se busca superar, a través de varias propuestas, las críticas recogidas por la planificación desarrollada con anterioridad. Una de las primeras soluciones es abandonar el "hipotético soporte medio" utilizado en las etapas anteriores, para observar lo que ha ocurrido exactamente con la inserción publicitaria en términos de impactos. La frecuencia sufre una transformación similar. Este periodo, en torno a la década de los 90, continúa en fase de discusión y desarrollo. Gran parte de los retos surgen ante la creciente penetración de las nuevas tecnologías. 
Por supuesto, los tres niveles no representan etapas claramente delimitadas pero sí permiten identificar el desarrollo sufrido por la cobertura y la frecuencia como resultado de la reflexión sobre la contribución de estos criterios a los objetivos publicitarios.

2.1. Primer nivel: la cobertura neta acumulada y la frecuencia. El objetivo de la maximización.

Durante esta etapa, que comienza en la década de los 60 , se detecta una elevada producción de modelos para la estimación de la cobertura. Buena parte de estos modelos, en especial los estocásticos, se basan en un hipotético soporte medio. La cobertura se refiere al número de personas que son alcanzadas, al menos, una vez, dada una combinación de soportes y número de inserciones publicitarias.

La frecuencia es otro de los conceptos clave en la planificación de medios. La frecuencia media es el número medio de veces que se alcanza a la población. También puede ser expresada como una distribución. En este caso proporciona más información que la media pues contribuye, al menos, a la posibilidad de estudiar los efectos que producen (por ejemplo, en términos de recuerdo) un número de exposiciones concreto (y no un número medio de exposiciones). Sin embargo, el uso de la frecuencia media fue más habitual durante las primeras etapas.

La cobertura y la frecuencia son los parámetros básicos de la planificación de medios. La relación entre la cobertura y la frecuencia es estrecha. Si se realiza una selección de soportes afines al público objetivo, llegará un momento que una nueva inserción recaiga en las mismas personas (González y Carrero, 2003), por lo que no se conseguirá más cobertura sino más frecuencia. A estos dos conceptos se les une la limitación del presupuesto y el objetivo de la rentabilidad.

En este punto, toma especial protagonismo la modelización a través del proceso de optimización en contraposición con los modelos de evaluación.

Los modelos de evaluación precisan de la previa combinación de soportes por parte del planificador quien debe proponer más de una posibilidad. El inconveniente que tienen estos modelos es que se desconoce si, además de los planes propuestos por el planificador, podría existir una combinación que sea mejor o que mejorara sustancialmente modificando levemente algún parámetro (por ejemplo, el presupuesto).

Este inconveniente es solventado por los modelos de optimización. La lógica que subyace en estos modelos es la extracción, con ayuda de un programa informático, de aquella combinación de soportes que maximiza la eficacia al mínimo gasto. Por tanto, estos modelos trabajan con dos funciones: la función de eficacia o rendimiento y la función de costes (Díez y Martín, 1999). 
Sin embargo, la primera limitación de la función de eficacia o rendimiento es lo que representa justamente la eficacia en estos modelos. Es decir, los objetivos del plan se circunscriben en obtener la máxima cobertura o una frecuencia de impactos determinada, dado un presupuesto. Y, por tanto, dado un presupuesto, el objetivo de maximización conduce a apostar por aquel plan que consiga más cobertura o más frecuencia sin, a priori, otro tipo de consideraciones.

Una segunda limitación es que los modelos de optimización no actúan en las dos funciones a la vez, sino que actúa sobre una restringiendo la otra. Es decir, otorgan la posibilidad bien de maximizar la eficacia (a través de la cobertura o la frecuencia) con un coste limitado, bien de minimizar el coste estableciendo un límite en la eficacia. Además, la principal desventaja que presentan estos modelos es su rigidez en la elección del criterio por el cual se pretende definir el rendimiento, es decir, se debe elegir entre maximizar la cobertura o maximizar la frecuencia (Díez y Martín, 1999).

2.2. Segundo nivel: cobertura y frecuencia efectiva. El objetivo de la optimización bajo el planteamiento de la respuesta favorable

La cobertura efectiva se refiere a las personas expuestas al anuncio de las que cabe esperar una respuesta positiva al estímulo publicitario (ie. Goodrich y Sissors, 1995; González y Carrero, 2003). En comunión con este término surge la frecuencia efectiva o el nivel de exposición al que se consigue este efecto. Desde esta postura parece necesaria una frecuencia de impacto para generar una respuesta. Pero, además, también se contempla la posibilidad de un umbral máximo de exposiciones, a partir del cual el efecto pretendido en el público puede volverse contra la campaña publicitaria o marca.

No obstante, la aplicación de la frecuencia efectiva tendrá muchas dificultades. La principal limitación viene de la mano, justamente, de la creencia extendida de que la persona deba necesariamente exponerse un número de veces mínimo al mensaje publicitario. De hecho, teorías posteriores, ya en la tercera etapa, se centrarán en conseguir impactar (una vez podría ser suficiente) "con la mayor proximidad posible al momento de la compra" (PérezLatre, 1999:62). Pero además, el establecimiento del número mínimo y máximo provoca versiones enfrentadas según el aspecto de la reacción del público que se esté midiendo (recuerdo, actitudes o intención de compra).

Con respecto al recuerdo y en lo que concierne a la planificación de medios, el umbral mínimo se establece en tres contactos en televisión (Krugman, 1972, 1977). Esta tesis defiende que una exposición menor de tres no surte un efecto positivo al no alcanzar el umbral para que se recuerde. De acuerdo con el clásico modelo de Krugman: la primera exposición genera en el individuo una respuesta sólo cognitiva, de reconocimiento e identificación, la segunda exposición permite completar el conocimiento sobre la naturaleza de dicha comunicación además de generar una respuesta evaluativa (establece juicios sobre 
el mensaje), finalmente, en la tercera exposición y a partir de la misma se empieza a generar recuerdo. Esta teoría se consideró una de las referencias fundamentales en materia de frecuencia de exposición.

La aplicación de esta teoría desemboca en una frecuencia media y no a un intervalo delimitado por un número mínimo y un número máximo de exposiciones. Así, el número tres aparece como el objetivo de frecuencia a alcanzar en términos medios. Pero, además, las tres exposiciones han sido utilizadas sin atender a las condiciones bajo las que el autor propuso el modelo, es decir, "dado un hipotético soporte medio", lo que no siempre se corresponde ni con el panorama mediático actual ni con las características específicas de cada medio.

Con respecto a las actitudes hacia el anuncio, los límites en términos de frecuencia de exposición pueden variar (Miquel, 1999). En términos generales se concluye que, del mismo modo que a mayor repetición crece el recuerdo también crece y mejora la actitud hacia el mensaje. La penetración del anuncio se manifiesta desde la primera exposición (quizás por la buena predisposición de los colaboradores de los estudios) y el desgaste empieza (en términos de actitud) a partir de la tercera exposición. En consecuencia, el umbral máximo sería de tres exposiciones. Los resultados no son concluyentes, entre otras causas porque las investigaciones que buscan proponer umbrales se basan en observaciones controladas, es decir, son estudios de laboratorio, en los que se suele utilizar grupos pequeños de personas predispuestas a colaborar.

Finalmente, con respecto al comportamiento tampoco hay resultados definitivos sobre la frecuencia precisa. Pese a la perspectiva DAGMAR y sus derivados, algunos estudios han demostrado en el ámbito de la comunicación comercial que no existe vinculación entre repetición e intención de compra (Miquel, 1999).

En definitiva, no parece existir una respuesta unívoca que permita establecer los umbrales de frecuencia. A todo ello se le debe añadir que los umbrales de frecuencia de estos estudios se extraen bajo el supuesto de estabilidad en otros factores que repercuten, sin duda alguna, en la eficacia de la publicidad.

Las principales limitaciones que tiene la aplicación del modelo de la cobertura y frecuencia efectivas se pueden resumir en las siguientes:

1. Tomar un valor en lugar de una distribución. Es decir, existe una gran simplificación a la hora de tomar el número tres, o cualquier valor, como nivel de exposición óptimo en términos de eficacia.

2. La falta de consenso. No existe consenso en términos del número máximo y mínimo para los resultados afectivos y conativos, incluso bajo el supuesto de que el resto de factores que influyen en la curva de respuesta se mantengan estables. 
3. El efecto de otros factores. En la práctica, no se suele establecer un número máximo universal en términos de frecuencia de exposición, por lo que se ignora el efecto desgaste de la campaña.

4. La falta de investigación. En estrecha relación con el anterior punto, cada campaña debería ser "probada" antes de su lanzamiento para conocer el umbral mínimo y máximo con el mensaje que se pretende transmitir. Pero además, es fundamental hacer una evaluación previa en términos de respuesta publicitaria para no cometer el error de atribuirle un efecto a la campaña que no le corresponde. Con todo, este último punto es más difícil de conseguir ya que depende de la rentabilidad de la investigación (Martín, 1999).

\subsection{Tercer nivel: la aplicación de los criterios en su justa media.}

La nueva fase iniciada en la década de los 90 se caracteriza por la observación de lo concreto, es decir, a través de la observación del soporte real (y no del hipotético soporte medio) y de una distribución de frecuencias que aporte información de cada nivel de exposición (y no de un conjunto de soportes). A estas propuestas se les une las voces que defienden la necesidad de, en definitiva, cambiar la sistemática de trabajo de la planificación.

En este caso también se tiende a partir del "recuerdo" como fase inicial o efecto mínimo a conseguir con la campaña publicitaria. De hecho, y de nuevo, estas propuestas no aportan conceptos significativamente distintos a los ya definidos por las teorías desarrolladas con anterioridad, salvo en el caso de la aplicación de la eficacia publicitaria en Internet. Con esta excepción, para el resto de medios publicitarios, se demanda la aplicación del conocimiento existente con la precisión necesaria, es decir, con las limitaciones que acompañaba a cada fase anterior y extrayendo aquellas aportaciones que contribuyen a la eficacia de la campaña publicitaria.

Para esta tercera etapa, se quiere destacar la propuesta de un grupo de investigadores (Cannon y Riordan, 1994; Cannon, Leckenby y Abernethy, 2002) como respuesta a las limitaciones expuestas. Ellos proponen lo que denominarán la Frecuency Value Planning (FVP) cuya explicación atiende a comprender este modelo como una forma de planificación a través de la probabilidad de exposición basada en la distribución de frecuencias.

El modelo FVP queda integrado en la estrategia de marketing y comunicación que establece los objetivos a conseguir con la campaña publicitaria y el presupuesto destinado a tal fin. Los autores proponen cuatro pasos para efectuar un plan de medios preocupado por ser eficaz:

- En primer lugar, seleccionar los soportes basándose en su alcance útil. Según los autores, esta propuesta es contraria a la que normalmente se aplica pues, los planificadores sumergidos en la búsqueda de las tres exposiciones por individuo, 
suelen terminar escogiendo aquellos soportes que generan mayor duplicación de audiencias.

- En segundo lugar, estimar la distribución de frecuencias teniendo en cuenta el efecto que un nuevo impacto genera en el anterior.

La distribución de frecuencias a la que se aludía en las etapas anteriores se estimaba para un conjunto de soportes. La modelización de la distribución utiliza la ley de la probabilidad y, por tanto, los OTS. Se apoya en un hipotético soporte medio y desarrolla el modelo matemático de estimación de la distribución de frecuencias aplicando, normalmente, la probabilidad de exposición a ese soporte a través del concepto Oportunidad de Ver.

La primera propuesta a destacar vinculada a este segundo paso se dirige a la estimación de las distribuciones atendiendo a los impactos actuales que causa la publicidad. Es decir, se utilizan frecuencias relativas basadas en el porcentaje de público objetivo que se expone habitualmente al soporte (la afinidad del soporte), considerando como soporte el nivel más específico y cercano a la forma publicitaria. Esta concreción del soporte es necesaria para reducir el nivel de incertidumbre en la exposición y, así, asegurarse de que la oportunidad de haber visto el anuncio (probabilidad) se acerca más al concepto de impacto (evidencia).

Hay dos limitaciones a esta propuesta. La primera es que, pese a aproximarse al concepto de impacto, se seguirá utilizando datos anteriores relativos al soporte para hacer predicciones, pues los impactos futuros son sencillamente imposibles de conocer. Por supuesto, esta limitación se supera con las técnicas y procedimientos habituales existentes para hacer estimaciones apropiadas (bien a través de la normalización.- mediante los estudios empíricos.- o a través de la modelización.- mediante modelos de regresión). En segundo lugar, conocer los impactos causados exactamente por la forma publicitaria (por la inserción) no siempre es posible ya que las fuentes que proporcionan estos datos tienen claras limitaciones para recoger este tipo de información, fundamentalmente cuando la medición se hace a través de encuestas. En este sentido la matización que implica la utilización del concepto OTS (Opportunity To See) en lugar del término "impactos" es acertada pues sólo se puede conocer si alguien ha tenido la oportunidad de haber visto el anuncio por haber estado expuesto al medio y al soporte en el que fue insertado. Es decir, por ejemplo, se pregunta si recuerda haber hojeado una revista, incluso un ejemplar, pero no se pregunta acerca de la publicidad. Esta limitación es mucho más difícil de superar.

Una segunda propuesta vinculada a este segundo paso sería la lógica con la que se construye la distribución. En lugar de calcular la distribución para todos los soportes elegidos, se pueden utilizar los modelos de agregación secuencial que consisten en la generación de tal distribución paso por paso (soporte por soporte o añadiendo sucesivamente nuevos instantes temporales). Esta distribución proporciona más información del efecto que causa los nuevos contactos sobre los existentes. Este tipo de distribuciones pueden ser facilitadas por Sofres A. M. 
- En tercer lugar, se estima la curva de respuesta para cada nivel de exposición que forma la distribución de las exposiciones publicitarias (y no para el conjunto). Para ello, los autores sugieren tener en cuenta los efectos que los contactos anteriores causan en los posteriores.

- Finalmente, en cuarto lugar, los autores proponen el cálculo de los valores de la frecuencia, cada nivel de exposición. Estos valores son extraídos multiplicando el porcentaje de público objetivo alcanzado por cada nivel de exposición (una, dos, tres, cuatro... veces) por el valor que obtiene la curva de respuesta en cada uno de esos niveles.

Por ejemplo, dada una combinación de soportes (o plan), tras el cálculo de la distribución de frecuencias se estima que el $40 \%$ de la población objetivo es alcanzada una vez (por un anuncio). Además, según el cálculo de la curva de respuesta, se observa que la probabilidad de comprensión (o recuerdo) del mensaje cuando la población es alcanzada una vez es de 0.25. El valor de la frecuencia para el primer nivel de exposición asciende al 10\%. Este valor se interpreta como: las personas de las que cabe esperar una respuesta favorable con la primera exposición dada una combinación de soportes (sería la cobertura efectiva para la primera exposición).

Como estos valores son extraídos para todas las exposiciones posibles, al final también se obtiene un número global, suma de los valores por niveles de exposición. El modelo propuesto tiene variantes para extraer los valores en relación a los GRP (gross rating point), al presupuesto disponible (lo que proporciona información sobre el coste por lo que lo llaman effective point) y se puede aplicar para conocer el beneficio en términos monetarios por la campaña propuesta.

La demanda de un procedimiento en planificación de medios más adecuado para conseguir eficacia, aportación que refleja la propuesta del modelo FVP, se encuentra en otras obras nacionales aunque no hagan alusión concretamente al FVP (i.e. Sánchez, 1999, Miguel, 2000, Pérez-Latre, 2000; De Toro y Ramas, 2000, González y Carrero, 2003).

En este periodo también se abre el debate para el medio Internet. Algunas aportaciones vienen de la mano de Hong y Leckenby (1998) o de Kim y Leckenby (2003), que tratan de comparar los resultados de diferentes modelos de planificación para Internet. Otros tratan de dar respuestas que pueden estar relacionadas con la eficacia y la planificación de los nuevos medios desde la investigación de audiencias (los trabajos de la IAB y de AIMC) 0 centrándose en las estrategias y formatos más eficaces.

\section{Software}

A lo largo de todo el periodo, fundamentalmente a partir de los años 60 en el ámbito profesional, se han ido desarrollando programas informáticos que ayudan al planificador de 
medios y contribuyen a establecer umbrales de eficacia. El software relacionado con la investigación y la planificación de medios que contribuye a estos propósitos ha sido llevado a cabo por diferentes organizaciones.

En primer lugar, las grandes agencias de publicidad contribuyen con programas propios que incluyen otros factores tales como la estacionalidad de las ventas para establecer el calendario de inserciones de una campaña (González y Carrero; 2003). En segundo lugar, existen empresas especializadas en este tipo de programas que venden sus servicios a las agencias de medios y de publicidad. El caso más sobresaliente es el de ODEC y su programa TOM micro, que facilita el análisis del panorama mediático y la realización de los planes de medios una vez cargados los datos de audiencias del EGM. Por último, las propias fuentes de información han desarrollado programas para gestionar su información (Info XXI de Infoadex y Infosys de Sofres A.M) y realizar optimizaciones (I nfosys de Sofres A.M.).

\section{Conclusiones}

La aportación de la planificación de medios a la eficacia significaba, en una primera etapa, maximizar la cobertura y el número de exposiciones. La controversia, en todo caso, emerge cuando la cantidad de presupuesto era limitado y no se podía maximizar ambos criterios. Esto se debe a que, dado un presupuesto, la cobertura y la frecuencia son dos estrategias con direcciones opuestas. Es decir, el aumento de la frecuencia de exposición implica alcanzar un número mayor de veces a las mismas personas (no a personas distintas.- que sería la lógica de la cobertura).

En un segundo nivel, la aportación radica en el establecimiento del valor óptimo de frecuencia a partir del cual se extrae la curva de respuesta y, de aquí, la distribución de contactos. Sin embargo, la contribución de la planificación a la eficacia publicitaria responde a la búsqueda de un valor o, a lo sumo, a un margen entre dos valores, que corresponda con la optimización, (maximización de la cobertura o de la frecuencia minimizando coste).

En esta tercera etapa, se toma como ejemplo el modelo FPV para destacar las principales aportaciones, algunas de ellas ya subrayadas por otros autores. La contribución de la planificación a la eficacia implica un cambio de sentido. Ya no se trata de encontrar un valor (máximo u óptimo) de cobertura y frecuencia, sino varios valores (más de dos) correspondientes al nivel de exposición del público objetivo desde el primer contacto hasta el último posible. Estos valores estarían centrados, en la medida de lo posible, en los impactos de la publicidad. Esta consideración le da también otro sentido a la optimización (por niveles de exposición) al relacionar dichos niveles con la cobertura alcanzada en los mismos, pero también otorga un nuevo protagonismo a la evaluación.

Como se ha expuesto, a partir de los trabajos pioneros en torno a la frecuencia de los planes de medios (Krugman, 1972; Naples, 1979; Jones, 1995 y Bogart, 1995) otros autores nacionales e internacionales se han preocupado y abordado esta cuestión. Ciertamente, 
encontrar una ecuación que permita averiguar la frecuencia media, su distribución y los intervalos a lo largo del tiempo más óptimos implica un control casi inabordable de multitud de variables que conforman el contexto mediático actual que, además, está en continuo cambio. Con todo, se pueden establecer pautas, encontrar las dificultades para un mayor control o descubrir cuáles serían los aspectos más relevantes que deberían estar incluidos en esa ecuación a través de la investigación.

De hecho, mientras que el ámbito profesional trata de dar respuestas a través del desarrollo de software que introduzca estas consideraciones y facilite la labor del planificador, el ámbito académico sigue denunciando las pocas investigaciones relacionadas con estas apreciaciones en todo el periodo revisado. Estudios centrados en cuantificar y valorar la producción científica en publicidad con repercusión internacional (i.e. Carlson, Grove, Stafford, 2005) apoyan esta conclusión. Se precisa más investigación sobre la contribución de la planificación de medios a la eficacia de la publicidad pero también sobre la conceptualización de los constructos, los medios de comunicación de masas e Internet, el impacto de la publicidad como una fuerza social y, en general, sobre todo lo concerniente al ámbito publicitario.

\section{REFERENCI AS BI BLI OGRÁFI CAS}

AAKER, David A. y Myers, John G. (1993): Management de la Publicidad. Perspectivas prácticas (Tomo II). Barcelona, Editorial Hispano Europea.

BeERLI, Asunción (1999): Técnicas de medición de la eficacia publicitaria. Barcelona, Ariel.

Bigné AlcaÑIZ, J. Enrique (1991a): “Problemática de la audiencia y de la publicidad ante los nuevos medios de comunicación", I nvestigación y Marketing, no 36, pp 15-27.

Bigné AlCAÑIZ, J. Enrique (1991b): “Innovaciones y nuevas tecnologías en investigación de audiencia y publicidad", Alta Dirección, n 157, mayo junio, pp 221-232.

BOGART, Leo (1995): "Is there an optimum frequency in advertising?", en Admap, febrero, pp 32-34.

CANnon, Hugh, LeCKenBy, Jonh D., y ABernethy, Avery (2002): “Beyond Effective Frecuency: Evaluating media Schedules Using Frecuency Value Plannig", en J ournal of Advertising Research, november, pp 33-47.

CANNON, Hugh. y RIORDAN, E. (1994): “Effective Reach and Frecuency: Does it Really Make Sense?", J ournal of Advertising Research, 34, 2, pp 19-28.

Carlson, L.; Grove, S. y Royne, M. (2005): "Perspectives on Advertising Research", en J ournal of Advertising, vol. 34, no 2, summer, pp 117-149.

De Toro, Juan Manuel y RAMAS, Santiago (2000): Mejorar la eficacia de la Publicidad en TV. Barcelona, Gestión 2000. 
Del Río Pereda, Pablo (2000): “No me chilles que no te veo. Atención y fragmentación audiovisual", en C\&E. Cultura y Educación, no 20, pp 51-80.

Díez De Castro, Enrique y Martín Armario, Enrique (1999): Planificación publicitaria. Madrid, Pirámide.

díez de Castro, Enrique, Martín Armario, Enrique y Sánchez franco, Manuel (2001): Comunicaciones de marketing. Planificación y Control. Madrid, Pirámide.

GonzÁlez Lobo, Mã Ángeles y CARRero LóPEZ, Enrique (2003): Manual de Planificación de Medios (4ạ Edición). Madrid, ESIC.

GoOdRICHI, William B. y SISSORS, Jack Z. (1995): Media Planning Workbook. Illinois, NTC Business Book.

HONG, John y LECKENBY, John D. (1998): “Using Reach/Frequency for Web Media Planning", en J ournal of Advertising Research, 38, 2, pp 7-20.

JONES, John P. (1990): “Advertising: Strong Force or Weak Force? Two Views an Ocean Apart", en International Jorunal of Advertising, no 9, pp 223-246.

J ONES, J ohn. P. (1995): When Ads Work: New Prof. That Advertisig Triggers Sales, Nueva York.

KIM, Hyo G. y LECKENBY, John D. (2003): “An IMC Planning Framework Using Reach and Frequency Concepts", (en línea) Working Paper

KRUGMAN, Herbert (1972): "How potent is television advertising? Some guidelines from theory" ANA Television Workshop Presentation, Nueva York, octubre.

KRUGMAN, Herbert (1972): “Why Three Exposures May Be Enough", Journal of Advertising Research, 12 (6), pp 11-14

KRUGman, Herbert (1977): “Memory without Recall, Exposure without Perception", Journal of Advertising Research, 17.4, pp 7-12

LAMAS, Carlos (1999): “La eficacia de los planes de medios: Apuntes para un modelo general", en 15 Seminario AEDEMO de Televisión, Bilbao, Febrero.

León, Jose Luis (1996): Los efectos de la Publicidad. Barcelona, Ariel Comunicación.

MARTín SANTANA, Josefa D. (1999): “Técnicas de control” en Sánchez Franco, Manuel J., Eficacia Publicitaria. Teoría y Práctica. Madrid, McGrawHill, pp 213-249.

MARTINEZ RAMOS, Enrique (1992): El uso de los medios de comunicación en marketing y publicidad. Madrid, Akal Comunicación.

MARTINEZ RAMOS, Enrique (1996): “Cómo trabaja la publicidad en el consumidor y la pérdida de su eficacia" en EGUIZÁBAL, Raúl y CARO, Antonio: Medición, investigación e información de la Publicidad. Madrid, Comunicación 2000, pp 85-112. 
Miquel Romero, Mạ José (1999): “La repetición del anuncio", en Sánchez Franco, Manuel J., Eficacia Publicitaria. Teoría y Práctica. Madrid, McGrawHill, pp 197-210.

Miquel Romero, Ma José (2000): “La creatividad en la planificación de medios: una vía de mejora de la eficacia publicitaria", en Bigné, J. Enrique, Temas de Investigación de Medios Publicitarios. Madrid, ESIC, pp 131-152.

NAPLES, Michael J. (1979): Effective Frequency: the Relationship between Frequency and Advertising Effectiveness. Nueva York, Association of National Advertisers.

Pérez latre, Francisco J. (2000): Planificación y gestión de medios publicitarios. Barcelona, Ariel Comunicación.

SÁnchez Franco, Manuel (1999): Eficacia Publicitaria. Teoría y práctica. Madrid, McGraw-Hill.

[Recibido: 30-11-2008. Aceptado, con necesidad de correcciones: 13-02-2009. Recibido con correcciones: 19-02-2009.] 